\title{
Disruption of microtubule network by Alzheimer abnormally hyperphosphorylated tau
}

\author{
Bin Li • Muhammad Omar Chohan • \\ Inge Grundke-Iqbal $\cdot$ Khalid Iqbal
}

Received: 27 December 2006 / Revised: 9 February 2007 / Accepted: 9 February 2007 / Published online: 20 March 2007

(C) Springer-Verlag 2007

\begin{abstract}
Hyperphosphorylated tau has long been proposed as the key molecule disrupting normal neuronal microtubule dynamics and leading to neurofibrillary degeneration in Alzheimer disease. Here we provide a direct evidence of hyperphosphorylated tau-induced disruption of microtubule network. Using Nocodozole-treated and detergent-extracted cells, we created a neuronal environment in mouse embryonic fibroblasts, 3T3 cells, by replacing their cytoplasm with adult rat brain cytosol. By recreating neuronal microtubule network in these cells, we were able to follow the effects of hyperphosphorylated tau on microtubule dynamics in real time. Whereas recombinant human brain tau promoted assembly and bundling of microtubules, abnormally hyperphosphorylated tau isolated from Alzheimer disease brain cytosol (AD P-tau) inhibited the assembly and disrupted preformed microtubule network by sequestering normal brain tau and MAP2. This breakdown of the microtubule network was reversed by treatment of the extracted cells with protein phosphatase-2A. This study, for the first time, provides direct mechanistic insights into the molecular basis of both axonal and dendritic neurodegeneration seen in Alzheimer disease.
\end{abstract}

Keywords Alzheimer disease $\cdot$ Retrograde neurodegeneration $\cdot$ Detergent extracted cells

B. Li · M. O. Chohan · I. Grundke-Iqbal · K. Iqbal $(\bowtie)$

Department of Neurochemistry,

New York State Institute for Basic Research

in Developmental Disabilities, 1050 Forest Hill Road,

Staten Island, NY 10314-6399, USA

e-mail: iqbalk@worldnet.att.net

\begin{abstract}
Abbreviations
$\mathrm{AD}$

MT

Alzheimer disease

MAP Microtubule associated protein

AD P-tau AD brain cytosolic abnormally

hyperphosphorylated tau
\end{abstract}

\section{Introduction}

Alzheimer's disease (AD) is characterized by profound central neurodegeneration. While it is a recurring theme in many neurological and neuro-psychiatric disorders, the precise mechanisms leading to the loss of neuronal mass have varying etiologies. In neurofibrillary degeneration of Alzheimer type, the affected neurons exhibit a particular type of pathology that evolves in a distal-to-proximal manner. This so-called, retrograde degeneration begins distally in the axo-dendritic network disrupting normal synaptic activity. The basis for retrograde degeneration can be explained by the presence of altered microtubule (MT) dynamics.

MTs play an essential role in maintaining the structural and physiological integrity of neurons. Neurodegenerative disorders, including $\mathrm{AD}$, show evidence of cytoskeletal dysfunction at both cytoarchitectural and molecular levels. Changes in neuronal shape, loss of dendrites and dendritic spines and abnormal accumulation of cytoskeletal proteins either in the form of inclusions like neurofibrillary tangles of paired helical filaments (PHF) seen in AD [24], or Lewy bodies seen in Parkinson's disease [29], constitute evidence of cytoarchitectural abnormalities. These neurons with anatomical aberrations show impaired function, which is seen as loss of synapses, decrease in synaptic conductivity and synaptic plasticity. This places the neuronal cytoskeleton as a key target for research and drug development. 
In the normal brain, MT assembly is stimulated by a number of microtubule-associated proteins (MAP), the high molecular weight MAP1 and MAP2, as well as the $50-75 \mathrm{kDa}$ tau proteins [37, 39]. It was previously reported by us that in $\mathrm{AD}$, tau is abnormally hyperphosphorylated and aggregated into bundles of paired helical filaments (PHFs), which progressively replace the neuronal cytoskeleton [20, 21]. In situ and in vitro observations suggest that the cytosolic/non-fibrillized abnormally hyperphosphorylated tau is inhibitory to MT assembly and stability $[1,2,3,25,31]$, whereas fibrillized tau is not [5, 9]. Moreover, only hyperphosphorylated tau, and neither MAP1 nor MAP2, has been shown to accumulate in the affected neurons in AD and related tauopathies. While it is desirable to study the effects of tau hyperphosphorylation on MT function in an in vivo system, there are several limitations surrounding direct and isolated manipulation of tau phosphorylation and its effect on MT dynamics in the living cell. Both genetic and pharmacologic means of studying mechanisms of neurofibrillary degeneration have several pitfalls. Transfected cell lines and transgenic animals overexpressing wild type, pseudophosphorylated or mutated tau do not reflect the pathology in AD in which neither any causative tau gene mutation nor increase in tau messenger RNA has been found [15, 32]. Modifying kinase and phosphatase levels/activities in the cell to achieve tau hyperphosphorylation [13] affects several other pathways in the cell $[6,10,12,14,23,40]$ and may not truly reflect the disease state. Consequently, the mechanisms proposed for the apparent toxicity of hyperphosphorylated tau have not yet been identified in the cell and therefore remain unestablished.

Detergent extraction of cells has been extensively employed in the literature to study components of the cytoskeleton [7]. When cells are treated with a non-ionic detergent, such as Triton X-100, approximately $70 \%$ of the cytosolic proteins are extracted [30]. Such detergentextracted cells retain many of the morphological features of the cytoskeleton seen in intact cells, including microfilaments, microtubules, intermediate filaments and associated proteins [16, 35].

Here, we describe a model in which we disrupted the MT network of 3T3 cells (mouse embryonic fibroblasts) with Nocodazole (Noco), followed by removal of the cytoplasm by extraction with Triton X-100 (Tri), and replaced the MT network of the host cells (Noco/Tri cells) with that polymerized from adult rat brain cytosol. The MT network formed from rat brain tubulin, MAP2 and tau created a neuron-like environment in which we could directly study the effects of AD P-tau in real time. We found that AD P-tau causes breakdown of MT network by sequestering both tau and MAP2. A compromised MT network both in the axons and dendrites might be the basis of the progressive retrograde degeneration of the affected neurons seen in $\mathrm{AD}$ and related tauopathies.

\section{Materials and methods}

Antibodies

The following primary antibodies were used: DM1A, (1:2000; Sigma, St Louis, MO, USA), a mouse monoclonal antibody to $\alpha$ tubulin; YL1/2, (Harlan Sera-Lab, Leicestershire, UK), a rat monoclonal antibody to tyrosinated/ dynamic-tubulin ; anti- Glu-tubulin, (1:500, a gift from A del C. Alonso), a rabbit polyclonal antibody to detyrosinated tubulin; 134d, (1:1000 for immunocytochemistry, 1:5000 for Western blots), a rabbit polyclonal antibody to total tau [11]; SMI52, (1:200; Sternberger Monoclonals, MD, USA), a mouse monoclonal antibody to MAP2; Tau1, (1:100, a gift from L. Binder), a mouse monoclonal antibody to un-phosphorylated tau; pSer 422, (1:100, raised in our laboratory), a rabbit polyclonal antibody to phosphorylated tau at serine 422; PHF-1, (1:5000, a gift from P. Davies), a mouse monoclonal antibody to phosphorylated tau at serine 396 and serine 404; M4, (1:2000, a gift from Y. Ihara), a mouse monoclonal antibody to phosphorylated tau at threonine 231 and serine 235; 12E8, (1:2000, a gift from D. Schenk), a mouse monoclonal antibody to phosphorylated tau at serine 262 and serine 356. The following secondary antibodies were used: Alexa 488-conjugated goat anti-mouse IgG antibody and Alexa 594-conjugated goat anti-rabbit or anti-rat IgG antibody (Molecular probes, Carlsbad, CA, USA); horseradish peroxidase-conjugated anti-mouse (Jackson ImmunoResearch, West Grove, PA, USA).

Preparation of rat brain cytosol

Adult Wistar rats (Charles River Laboratories, Wilmington, MA, USA), 3-5 months old, were used to prepare $61,000 \mathrm{~g}$ brain cytosolic extract as described previously [4]. Briefly, the brain was homogenized in equal volume $(w / v)$ of chilled PEM buffer (100 mM PIPES, $1 \mathrm{mM}$ EGTA, $2 \mathrm{mM} \mathrm{MgCl}_{2}$ at $\mathrm{pH}$ 6.9), containing a cocktail of protease inhibitors ( $4 \mu \mathrm{g} / \mathrm{ml}$ Aprotinine, $4 \mu \mathrm{g} / \mathrm{ml}$ Leupeptin, and $1 \mu \mathrm{g} / \mathrm{ml}$ Pepstatin). The homogenate was centrifuged at $61,000 \mathrm{~g}$ at $4^{\circ} \mathrm{C}$ for $30 \mathrm{~min}$ and the supernatant was referred as the cytosol.

\section{Preparation of Noco/Tri 3T3 cells}

Mouse embryonic fibroblast (3T3) cells were plated on poly-D-lysine coated 8-well culture slides (BD Biosciences, Bedford, MA, USA) and cultured in DMEM supplemented with $10 \%$ neonatal calf serum (both from 
Invitrogen, Carlsbad, CA). Upon reaching $\sim 50 \%$ confluence, the cells were washed in PEM buffer and either extracted with $0.2 \%$ Triton $\mathrm{X}-100$ for $1 \mathrm{~min}$ at $37^{\circ} \mathrm{C}$ or pretreated with $10 \mu \mathrm{M}$ nocodazole (ICN Pharmaceuticals, Casta Mesa, CA, USA) at $37^{\circ} \mathrm{C}$ for $4 \mathrm{~h}$ to depolymerize endogenous MTs before detergent extraction. The extracted cells, thus obtained were referred to as Noco/Tri cells.

Isolation of recombinant normal human tau and AD P-tau

Recombinant human brain tau $\mathrm{u}_{410}$ was expressed in the prokaryotic expression vector pRK172 and was purified as described previously [36], except that the perchloric acid extraction was avoided. AD P-tau was isolated from frozen autopsied AD brains, obtained within $6 \mathrm{~h}$ postmortem, as described [26] and further purified by Sepharose G-column chromatography (Amersham Bioscience, Arlington Heights, IL, USA).

\section{Microtubule Assembly in Noco/Tri cells}

Noco/Tri cells were rinsed with PEM buffer, and incubated with rat brain cytosol in the presence of $1 \mathrm{mM} \mathrm{GTP}$ at $37^{\circ} \mathrm{C}$ to study microtubule dynamics. Where indicated, recombinant $\operatorname{tau}_{410}$ or $\mathrm{AD}$ P-tau $(100 \mu \mathrm{g} / \mathrm{ml})$ was added and the extracted cells were analyzed for MT assembly by immunocytochemistry.

Dephosphorylation of AD P-tau by protein phosphatase-2A

Dephosphorylation of AD P-tau was carried out as described previously $[19,38]$. Briefly, AD P-tau $(0.2 \mathrm{mg} /$ $\mathrm{ml}$ ) was incubated in a mixture of $50 \mathrm{mM}$ Tris, $\mathrm{pH} 7.0$, $20 \mathrm{mM} \beta$-mercaptoethanol, $0.1 \mathrm{mg} / \mathrm{ml}$ bovine serum albumin, $1.0 \mathrm{mM} \mathrm{MnCl}{ }_{2}, 10 \mu \mathrm{g} / \mathrm{ml}$ each of leupeptin, pepstatin and aprotinin, and $1.0 \mathrm{U} / \mathrm{ml}$ protein phosphatase-2A at $37^{\circ} \mathrm{C}$ for $45 \mathrm{~min}$. Protein phosphatase-2A holoenzyme was purified from bovine brain as described previously [38]. For Western blot analysis, the reaction was stopped by the addition of $2 \times$ SDS-PAGE sample buffer and subjected to SDS-polyacrylamide gel electrophoresis. The blots were developed with the primary antibodies 134d, Tau-1, PHF-1, M4 and 12E8, followed by incubation with anti-mouse $\operatorname{IgG}$ conjugated with horseradish peroxidase and ECL detection reagents (Amersham Biosciences Corp., Piscataway, NJ).

\section{Immunocytochemistry}

After treatment, the extracted cells were fixed in a mixture of $0.3 \%$ glutaraldehyde and $0.5 \%$ NP-40 in PEM buffer at $37^{\circ} \mathrm{C}$ for $10 \mathrm{~min}$, washed with PBS and treated with $10 \mathrm{mg} /$ $\mathrm{ml}$ sodium borohydride (Sigma-Aldrich, St Louis, MO) for $7 \mathrm{~min}$. The extracted cells were then incubated with $0.1 \mathrm{M}$ glycine in PBS for $20 \mathrm{~min}$, washed with PBS and blocked with $4 \%$ horse serum for $1 \mathrm{~h}$ at $37^{\circ} \mathrm{C}$. The cells were incubated with the appropriate primary antibodies overnight at $4^{\circ} \mathrm{C}$. Next morning, after extensive washing with PBS, the cells were incubated in the blocking solution for $30 \mathrm{~min}$ at room temperature and then incubated with Alexa 488-conjugated anti-mouse antibody and Alexa 594-conjugated anti-rabbit or anti-rat antibody simultaneously, each at a final dilution of $1: 1000$, for $1 \mathrm{~h}$ at room temperature. Images were captured with Nikon PCM 2000 Confocal Imaging System.

Analysis of microtubule assembly by Western blot

Rat brain cytosol (15\%) was incubated in $100 \mu \mathrm{l}$ of PEM buffer containing $1 \mathrm{mM} \mathrm{GTP}$ at $37^{\circ} \mathrm{C}$ for $1 \mathrm{~h}$ in the presence or absence of normal or AD P-tau. The reaction mixture was then centrifuged at $5,600 \mathrm{~g}$, at $37^{\circ} \mathrm{C}$ for $10 \mathrm{~min}$ to separate assembled MTs. The supernatant and the MT pellets were then lysed in $1 \%$ sodium dodecyl sulfate (SDS) and $1 \%$ $\beta$-mercaptoethanol, followed by bath sonication and boiling for $5 \mathrm{~min}$. Indicated volumes of supernatant and dissolved pellets were subjected to SDS-polyacrylamide gel electrophoresis and employed for Western blots on PVDF membranes as described previously [20]. The blots were developed with the primary antibody anti- $\alpha$ tubulin (DM1A), followed by incubation with anti-mouse IgG conjugated with horseradish peroxidase and ECL detection reagents (Amersham Biosciences Corp., Piscataway, NJ).

\section{Results}

MT assembly in Noco/Tri cells

We employed 3T3 cells to study microtubule network assembly in intact cells and after extraction with Triton $\mathrm{X}-100$. Compared to the intact cells (Fig. 1a-c), Tritonextracted cells displayed remarkable preservation of the microtubule network (Fig. 1d). As previously reported [8], the MT in intact cells mainly contained tyrosinated (Tyr) tubulin, a form of tubulin found predominantly in dynamic assemblies of MT. We used detergent extraction in Nocodazole treated 3T3 cells to obtain a relevant "platform" on which to study MT dynamics in the cellular context. After extraction with Triton X-100, majority of the unpolymerized tubulin was removed, while some free tubulin could be seen attached to the detergent-resistant platform of the Noco/Tri cells (Fig. 1f). Next, rat-brain cytosol was used as a source of tubulin to form MT on Noco/Tri cells (Fig. 1gk). Only minimal MT assembly was observed at concentrations of $5-10 \%$ cytosol (Fig. 1g, h), whereas MTs readily assembled in the presence of higher concentrations of the 
Fig. 1 Microtubule assembly in Noco/Tri cells. 3T3 cells were extracted with $0.2 \%$ Triton X100 and used as a "cellular platform" for MT assembly. a-c Microtubule network in intact $3 T 3$ cells. $\mathbf{d}-\mathbf{f}$ detergent extracted cells with or without pretreatment with Nocodazole. MT structure is faithfully preserved in detergent extracted 3T3 cells (d). Pretreatment with Nocodazole destroys endogenous MT (e) and its components are largely lost on detergent extraction (f). Rat brain cytosol, at various concentrations $(5,10,15,25$ or $50 \%$ ) was added to Nocodazole pre-treated, detergent extracted $3 T 3$ cells (Noco/Tri cells) in the presence of $1 \mathrm{mM}$ GTP to study MT assembly $(\mathbf{g}-\mathbf{k})$. Nocodazole inhibited MT assembly when added to the rat brain cytosol on Noco/Tri cells (I). Alpha tubulin (DM1A, green), tyrosinated tubulin (YL1/2, red). Scale bar represents $25 \mu \mathrm{m}$
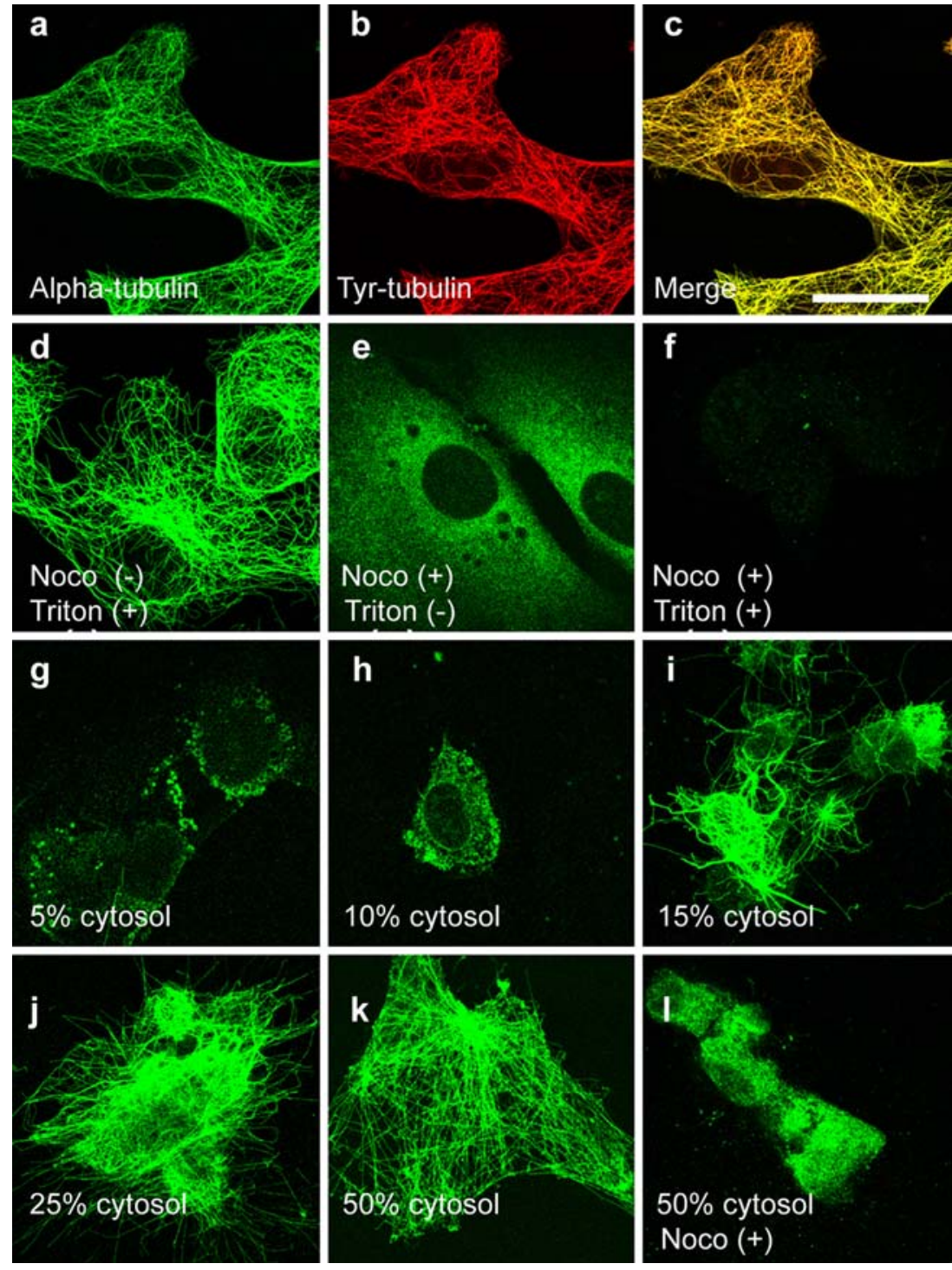

extract (Fig. 1i-k). Furthermore, similar to intact cells, Nocodazole treatment inhibited MT assembly in Noco/Tri cells and free tubulin could be seen attached to the Noco/ Tri cells (Fig. 11).

To investigate the dynamic course of MTs in Noco/Tri 3T3 cells, the time course of MT assembly was studied at different time intervals (from $1 \mathrm{~min}$ to $4 \mathrm{~h}$ ) after incubation of the extracted cells with $25 \%$ rat brain cytosol in the presence of $1 \mathrm{mM} \mathrm{GTP}$ at $37^{\circ} \mathrm{C}$. Microtubule-organizing center (MTOC)-like structures were evident as early as $1 \mathrm{~min}$ post incubation. Assembled MTs were observed growing from MTOC-like structures by $10 \mathrm{~min}$ and were stable up to $4 \mathrm{~h}$ (Fig. 2a).

To study the time course of MT disassembly, MTs were first assembled in Noco/Tri cells in the presence of $25 \%$ rat brain extract containing $1 \mathrm{mM}$ GTP for $20 \mathrm{~min}$. The extracted cells were then washed and incubated in PEM buffer, and fixed at different time intervals to study the time course of disassembly. Almost all MTs depolymerized by $1 \mathrm{~h}$ in this experimental paradigm (Fig. 2b).

Tubulin undergoes a unique post-translational modification in which a tyrosine is either attached to its C-terminal (glutamate) by a specific tubulin tyrosine ligase or is removed by tubulin carboxypeptidase. The generation of Glu-tubulin is a post-polymerization event in which detyrosination of some Tyr-MT forms Glu-MT as early as 20-25 min of polymerization. Consequently, Glu-tubulin predominates in stable assemblies of MTs present in differentiated cells such as neurons [22]. More than $75 \%$ of $\alpha$ tubulin in the adult mammalian brain is believed to be Glu-tubulin [33]. We found that Glu-tubulin represented the major tubulin component of assembled MT in Noco/Tri cells (Fig. 3a). MAPs are known to differ in their abilities to stimulate MT assembly; MAP2 promotes higher rates and higher levels of MT assembly and nucleation than tau [34]. We found that MAP2 predominated in assembled MT, with 
Fig. 2 Time course of MT assembly/disassembly in Noco/ Tri cells. 3T3 cells were pretreated with $10 \mu \mathrm{M}$ nocodazole prior to detergent extraction. Rat brain cytosol (25\%) was then applied to the Noco/Tri cells in the presence of GTP to study MT assembly at various time points (a). Microtubule organizing center (MTOC)-like structures could be detected as early as 1 min after incubation with adult rat brain cytosol, whereas by $10 \mathrm{~min}, \mathrm{MT}$ growth from MTOC could be observed. Assembled MTs were stable for up to $4 \mathrm{~h}$ studied. b To study disassembly, MTs were first assembled in Noco/Tri cells as described above for $20 \mathrm{~min}$. The cells were then washed, incubated in PEM buffer, fixed at different time points, immunostained and analyzed by confocal microscopy. By $1 \mathrm{~h}$, almost all MTs were disassembled. Anti-alpha tubulin (DM1A) was used for immunocytochemical staining. Scale bar represents $25 \mu \mathrm{m}$ a
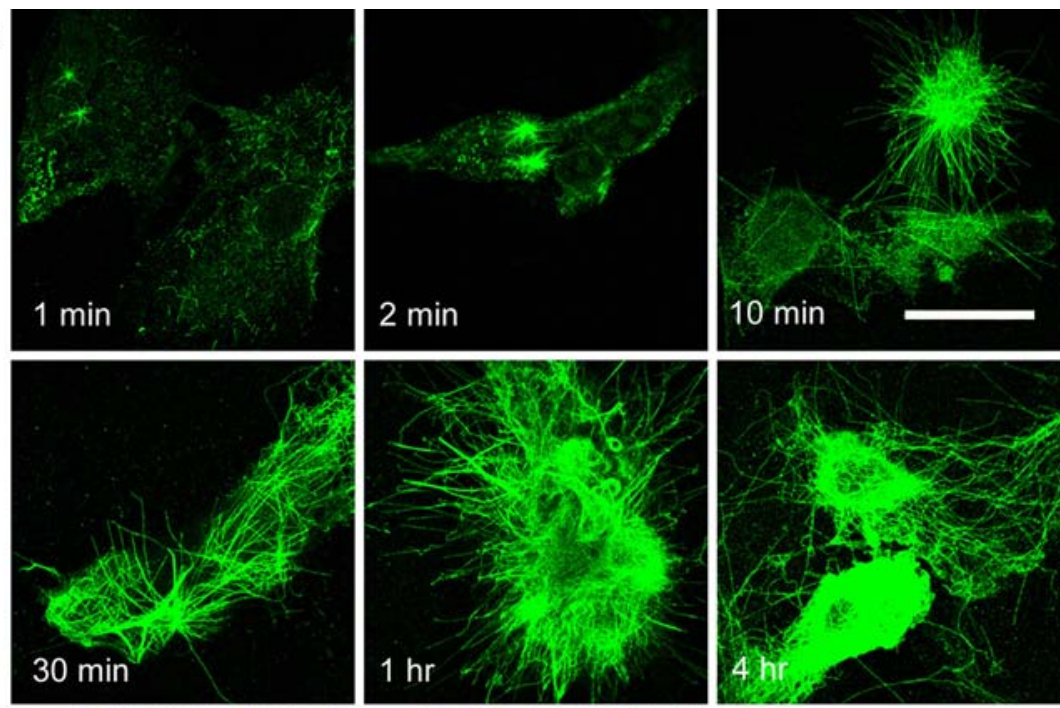

b
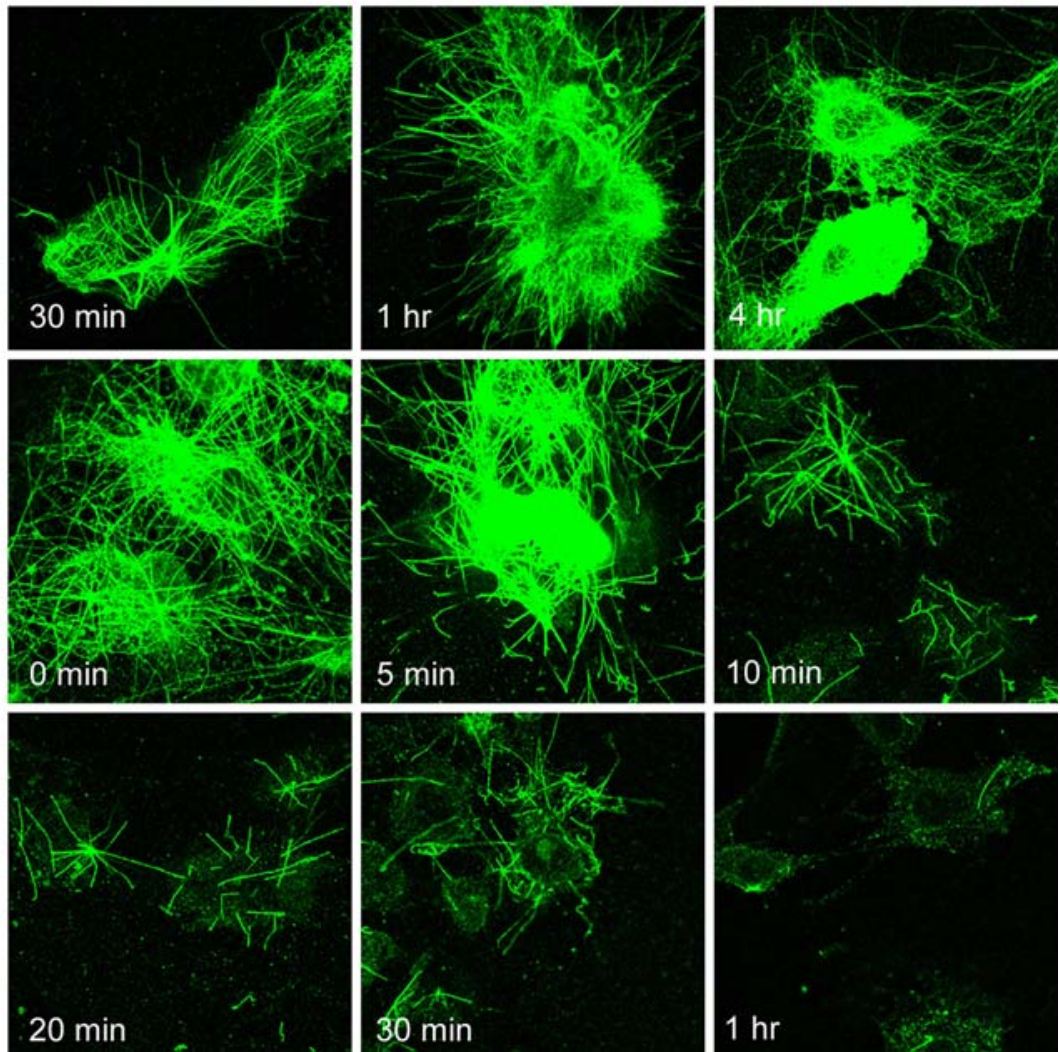

tau co-staining with relatively fewer MT in Noco/Tri cells (Fig. 3b).

\section{Effect of AD P-tau on MT assembly}

To study the effect of AD P-tau on MT network, we added AD P-tau or as a control recombinant tau ${ }_{410}(100 \mu \mathrm{g} / \mathrm{ml}$ each) to rat brain cytosol and incubated Noco/Tri cells with the reaction mixture for $1 \mathrm{~h}$ at $37^{\circ} \mathrm{C}$ in the presence of phosphatase inhibitors (100 $\mathrm{nM}$ okadaic acid and $25 \mathrm{mM}$ sodium floride). No MTs assembled in the presence of AD P-tau, and tau could be seen attached to the Noco/Tri cells (Fig. 4a). Recombinant tau on the other hand, promoted the bundling of MTs (Fig. 4a). These results suggest that AD P-tau inhibits MT assembly in Noco/Tri cells and that excess of normal tau (beyond that present in the $25 \%$ brain cytosol) causes bundling of MT.

Next, we investigated the ability of AD P- tau to breakdown preassembled MTs in Noco/Tri cells (Fig. 4b). After inducing MT assembly with rat brain cytosol for $20 \mathrm{~min}$, rat brain cytosol was replaced by an equal volume of either warm PEM buffer containing $1 \mathrm{mM} \mathrm{GTP}$ alone (control), or together with recombinant $\operatorname{tau}_{410}(100 \mu \mathrm{g} / \mathrm{ml})$, or AD P-tau $(100 \mu \mathrm{g} / \mathrm{ml})$, and incubated for $40 \mathrm{~min}$. AD P-tau, caused breakdown of MTs whereas recombinant tau promoted MT bundling.

\section{Binding of AD P-tau to normal MAPs in Noco/Tri cells}

In order to investigate whether the breakdown of MT network by AD P-tau induced its interaction with normal MAPs, we immunostained Noco/Tri cells, which were treated similar to Fig. 4a, with specific antibodies against un-phosphorylated, normal tau (Tau-1) and AD P-tau (pSer 422). Control and recombinant tau $\mathrm{t}_{410}$-treated Noco/Tri cells showed robust staining of MT with Tau-1 indicating that the majority of normal tau associated with MTs (compare with Fig. 4a). AD P-tau-treated Noco/Tri cells displayed 
Fig. 3 Characterization of MTs assembled in Noco/Tri cells. MTs were assembled in Noco/ Tri cells using $25 \%$ rat brain extract. The cells were then stained for modified alpha tubulin and various MAPS. a Majority of assembled MTs had detyrosinated (Glu-tubulin) tubulin ( $\alpha$-tubulin, green; Glu-tubulin, red). Tyrosinated tubulin was largely absent from assembled MTs (Tyr-tubulin, red). b MAP2 was the major MAP in assembled MTs whereas tau co-localized considerably less with single MTs. MAP2 green, tau red. Scale bar represents $25 \mu \mathrm{m}$ a
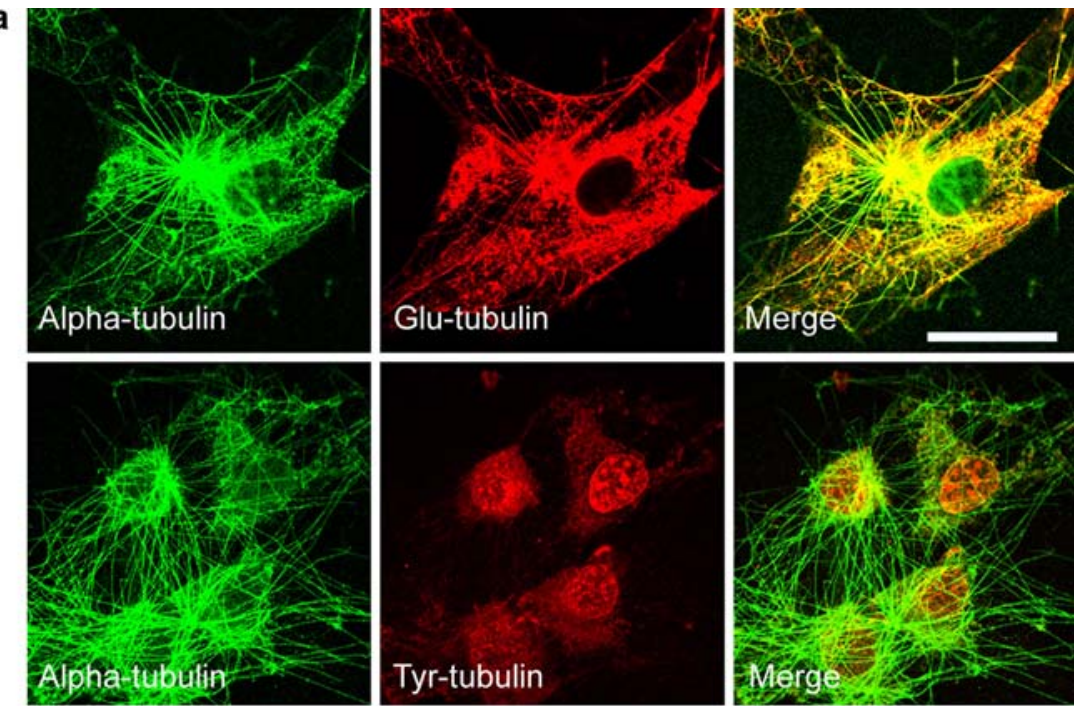

b
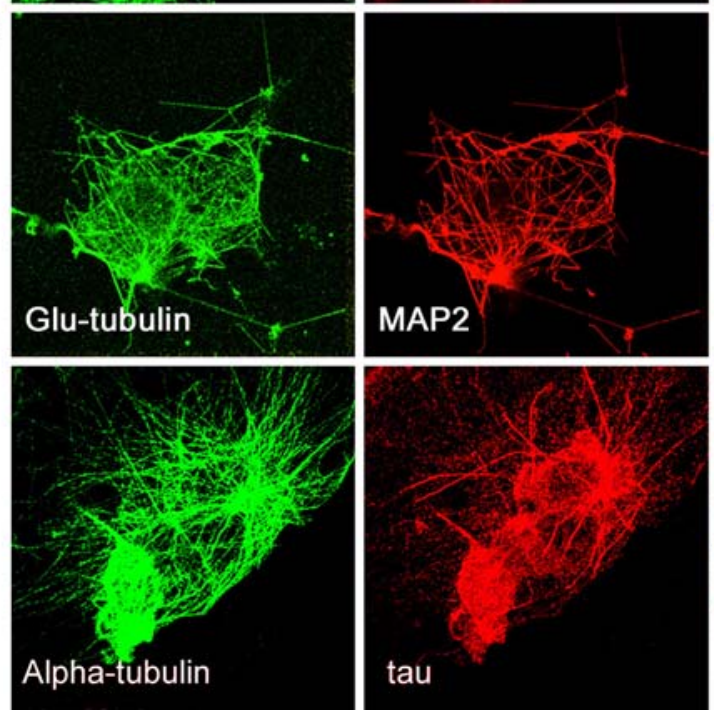
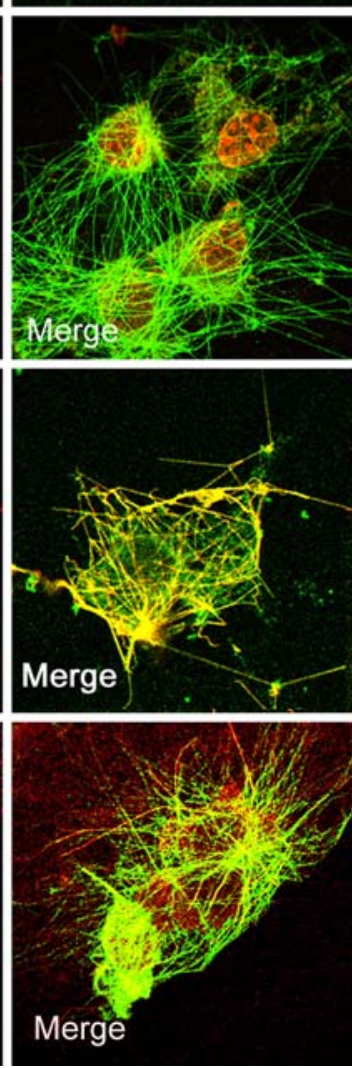

remarkable co-localization of Tau-1 with pSer422 indicating that AD P-tau had sequestered normal tau from the participating pool of MAPs (Fig. 5a). Similarly, AD P-tau also co-stained with MAP2 (Fig. 5b), indicating that it sequestered MAP2 from the system. Together, these results indicate that AD P-tau causes breakdown of MT network by sequestering normal tau and MAP2.

In order to find out whether the effect of AD P-tau on MT dynamics was dependent on its concentration, we next confirmed the effects of AD P-tau on MT assembly using Western blots. MT assembly was studied in vitro by incubating rat brain cytosol with varying concentrations (6.25$100 \mu \mathrm{g} / \mathrm{ml}$ ) of recombinant $t_{410}$ or AD P-tau. After incubation for $1 \mathrm{~h}$, the sample was centrifuged and supernatant and pellet were analyzed by Western blots to compare the amount of tubulin in both fractions (Fig. 6). The proportion of tubulin in the pellet reflected its assembly into MTs. About $60 \%$ of tubulin assembled into MTs in $15 \%$ rat brain cytosol. AD P-tau decreased the assembly of MTs in a dose-dependent manner with concentrations of $50 \mu \mathrm{g} / \mathrm{ml}$ and above totally abolishing MT assembly. Tau 410 by itself had no significant effect on MT assembly.

Dephosphorylation of AD P-tau by PP-2A restores normal MT dynamics in Noco/Tri cells

Tau is a phospho protein and its biological activity is regulated by the degree of phosphorylation [27]. Hyperphosphorylation alone is enough to confer tau with inhibitory activities towards MT assembly. The activities of protein kinases and phosphatases are therefore, critical to the regulation of tau phosphorylation. We have previously shown that AD P-tau can be dephosphorylated by protein phosphatases [17-19] and that this treatment restores its biological activity in vitro [38]. Protein phosphatase 2A, which is present in the brain and is relevant to AD pathology [28], has the highest efficiency of restoring MT assembly-promoting activity of AD P-tau in vitro [38]. We next studied 


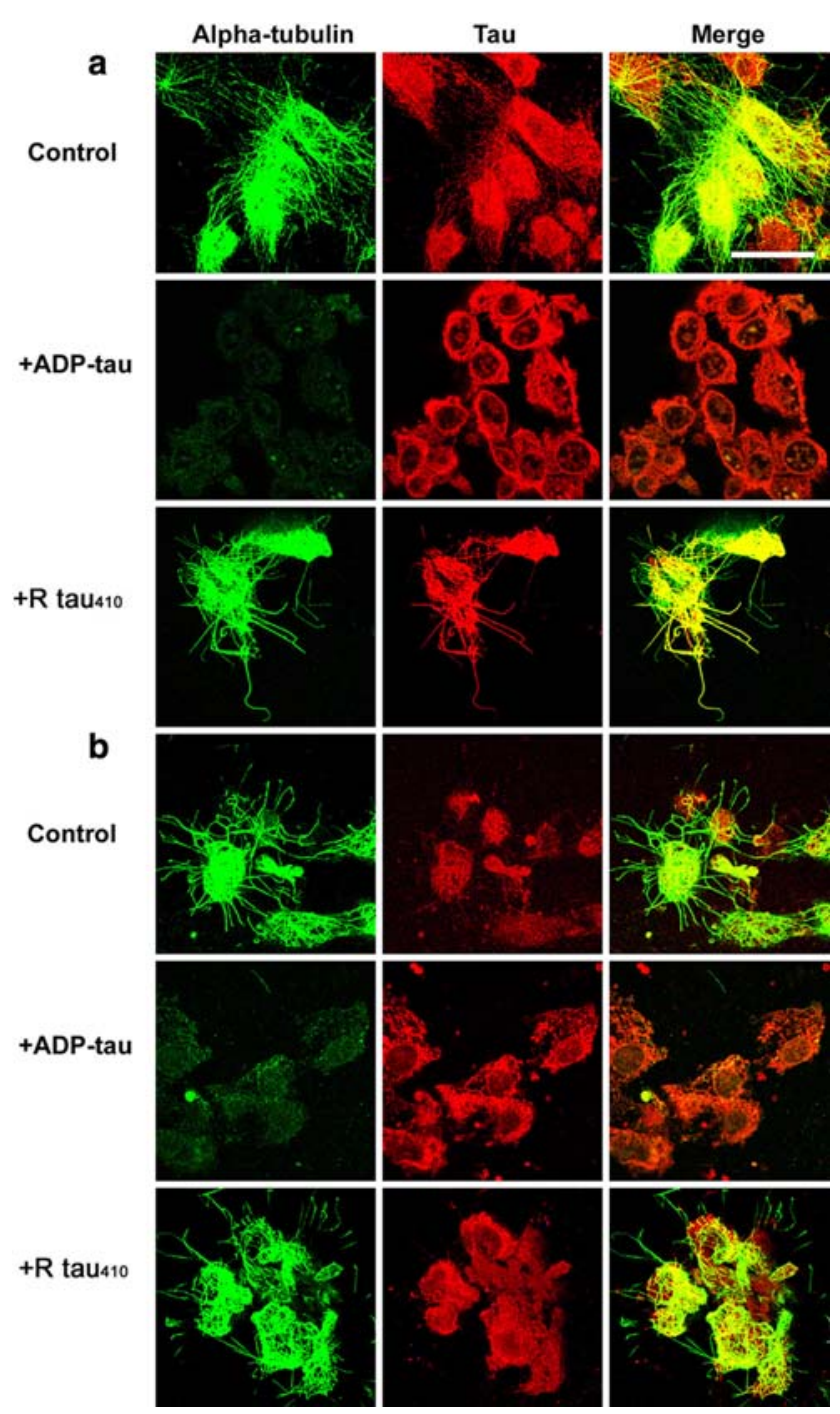

Fig. 4 Effect of AD P-tau on MT dynamics in Noco/Tri cells. MT assembly was induced with $25 \%$ adult rat brain extract in Noco/Tri cells in the presence or absence of $100 \mu \mathrm{g} / \mathrm{ml}$ of either recombinant human brain $\operatorname{tau}_{410}$ or AD P-tau. a AD P-tau, but not tau ${ }_{410}$, completely inhibited MT assembly. Tau 410 promoted assembly and bundling of MT. b MT were assembled on Noco/Tri cells for 20 min after which rat brain cytosol was replaced with either warm PEM buffer containing $1 \mathrm{mM}$ GTP alone (control), or $100 \mu \mathrm{ml}$ of tau ${ }_{410}$ or AD P-tau and incubated for $40 \mathrm{~min}$. AD P-tau, but not tau 410 , destroyed assembled MTs. Scale bar represents $25 \mu \mathrm{m}$. ( $\alpha$-tubulin green, total tau red)

whether treatment of AD P-tau with PP-2A can counteract its inhibitory activity towards MT assembly in Noco/Tri cells. For this purpose, we did two sets of experiments: in the first set of experiments, AD P-tau was treated with PP$2 \mathrm{~A}$ for $45 \mathrm{~min}$ at $37^{\circ} \mathrm{C}$ prior to its incubation with rat brain cytosol for MT assembly in Noco/Tri cells. PP-2A releases $\sim 50 \%$ of inorganic phosphate from AD P-tau within $45 \mathrm{~min}$ [38]. So the incubation mixture for MT assembly contained partially dephosphorylated AD P-tau. Treatment of AD P-tau with PP-2A partially restored its activity towards MT assembly (Fig. 7a-c).

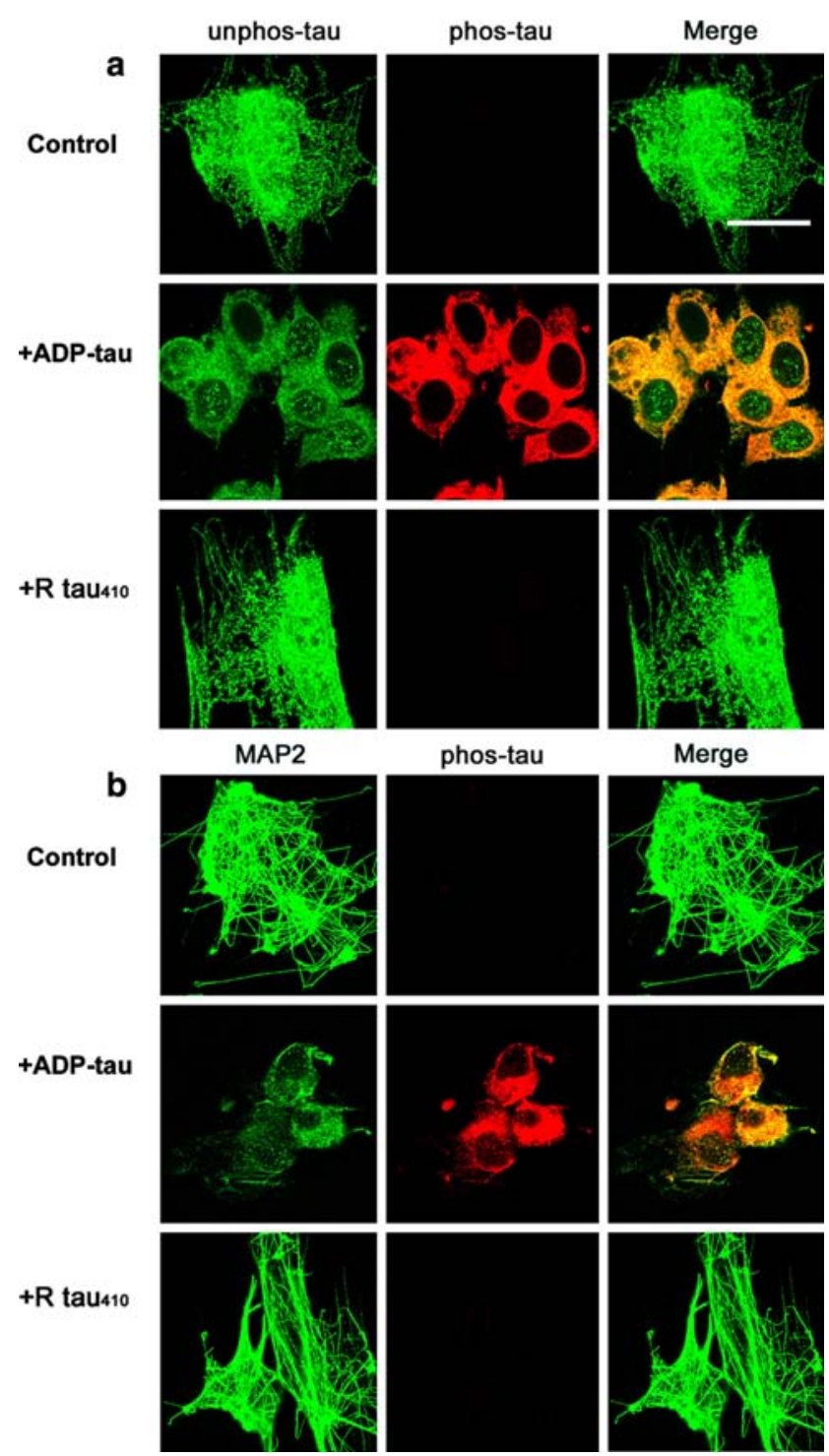

Fig. 5 Co-localization of AD P-tau with normal tau and MAP2 in Noco/Tri cells. MT assembly was induced with $25 \%$ adult rat brain extract in Noco/Tri cells in the presence or absence of $100 \mu \mathrm{g} / \mathrm{ml}$ of either recombinant human brain tau 410 or AD P-tau. AD P-tau sequestered normal tau and MAP2 as seen by co-localization of AD P-tau (stained with antibody to pSer 422, a site only phosphorylated in AD P-tau) with normal, unphosphorylated tau (a) and MAP2 (b). Scale bar represents $25 \mu \mathrm{m}$

In the second set of experiments, AD P-tau was first added to the rat brain cytosol in order to inhibit MT assembly in Noco/Tri cells (Fig. 7d). After 10 min, PP-2A was added to the Noco/Tri cells and the samples were incubated for another $20 \mathrm{~min}$. PP-2A activity was then inhibited by adding phosphatase inhibitors (100 $\mathrm{nM}$ Okadaic acid and $25 \mathrm{mM} \mathrm{NaF}$ ) and the cells were further incubated for $20 \mathrm{~min}$ in the presence of fresh GTP and thereafter fixed. A twenty-minute treatment of AD P-tau by PP-2A releases $\sim 30 \%$ phosphate per mole of AD P-tau [38]. There was clear evidence of at least some MT "re-assembly" as seen 

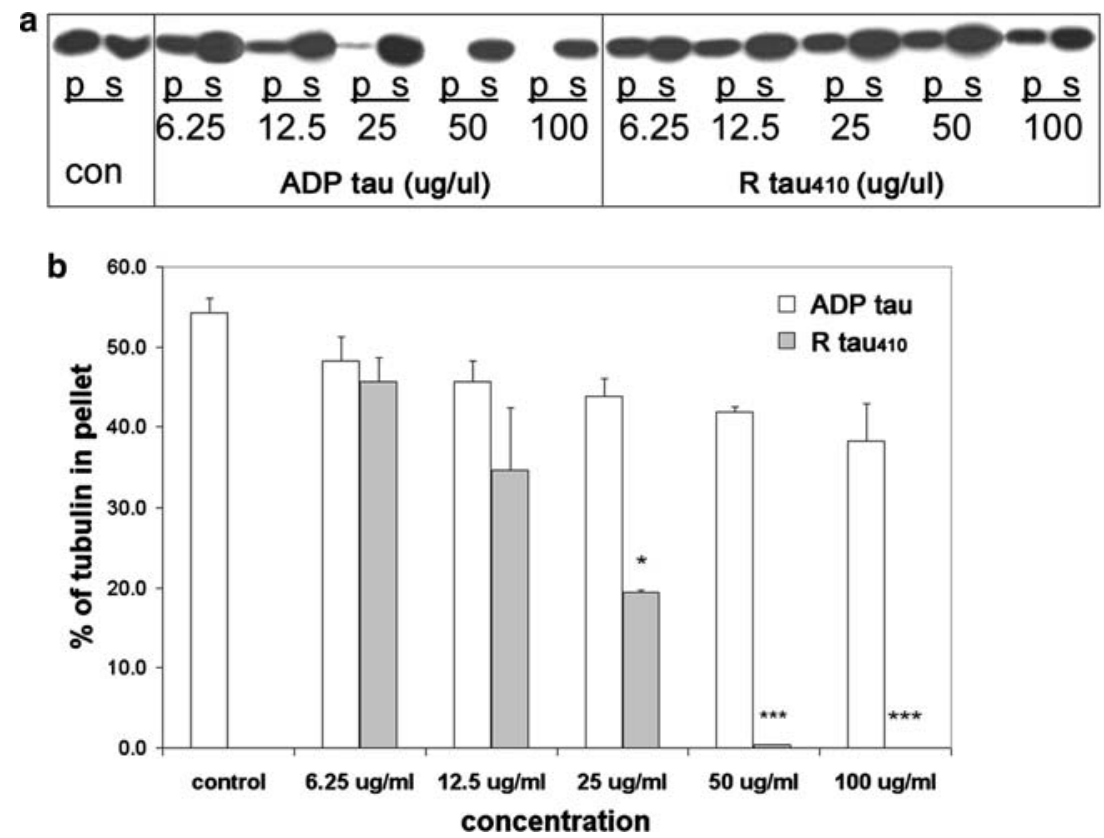

Fig. 6 Concentration-dependent effect of AD P-tau on MT assembly from rat brain cytosol. Fifteen percent rat brain cytosol containing $1 \mathrm{mM}$ GTP was used as a source of tubulin and MAPs for MT assembly. Recombinant tau $\mathrm{t}_{410}$ or AD P-tau was added at different concentrations $(6.25-100 \mu \mathrm{g} / \mathrm{ml})$ and incubated for $1 \mathrm{~h}$ at $37^{\circ} \mathrm{C}$. Pellets and supernatants were separated by centrifugation at 5,600g. a After dis-

by tubulin staining of the Noco/Tri cells (Fig. 7d-f). The extent of MT assembly in this set of experiments was less than that in the first set of experiments probably because of a relatively less dephosphorylation of AD P-tau.

Western blot analysis of AD P-tau pretreated with PP$2 \mathrm{~A}$ for $45 \mathrm{~min}$ at $37^{\circ} \mathrm{C}$, showed its dephosphorylation at multiple phosphorylation sites, as evident by immunostaining with Tau-1, PHF-1, M4 and 12E8 phospho-dependent tau antibodies (Fig. 7g).

These results indicate that AD P-tau competes with normal tau to inhibit MT assembly and that PP-2A-induced dephosphorylation of AD P-tau restores it to a normal-like protein.

\section{Discussion}

Dysregulation of MT dynamics leads to retrograde degeneration of neurons in Alzheimer disease and related tauopathies. With a disrupted MT network, many subcellular processes, which are vital for neuronal physiology are shut down, synapses are lost and the affected neurons die. One of the key elements in retrograde degeneration of Alzheimer type is the hyperphosphorylation of tau. Observations from in vitro studies suggest that hyperphosphorylated tau is inhibitory to MT assembly and stability. However, the precise mechanism by which hyperphosphorylated tau solving pellets in $0.4 \%$ SDS, both supernatants and pellets were analyzed with anti-tubulin (DM1A) by Western blots. b Quantitations were done by scanning chemiluminiscent (ECL) Western blots. AD Ptau decreased MT assembly in a dose dependent manner, whereas $\operatorname{tau}_{410}$ had no significant effect. Data in b represent mean \pm SEM. $* P<0.05, * * P<0.01, * * * P<0.001$

affects MT dynamics in the cell has been difficult to study. This is due to limitations in carrying out such studies without disturbing other cellular processes. In this study, we have employed a model in which we have replaced the cytoplasm of mouse embryonic fibroblasts with adult rat brain cytosol, creating a mature neuron-like environment of MT network made up of mostly Glu-tubulin, MAP2 and tau. Using this model, we show that AD P-tau causes a breakdown of the MT network by sequestering normal tau and MAP2, and that this inhibitory activity of AD P-tau can be reversed by its dephosphorylation by PP-2A.

Detergent extraction of cells with a non-ionic detergent like Triton X-100 removes all "soluble components" of the cytoplasmic ground substance. What remain are the elements of the cytoskeleton and the nuclear remnant. When intact cells are treated with Nocodazole to disassemble existing MT network, free tubulin is extracted along with the cytoplasmic ground substance. The remaining detergent extracted cells can now be employed as a "cellular platform" to create different cellular environments. By replacing cytosolic ground substance of the host cells with adult rat brain cytosol, a neuron-like environment is created in which the assembled MT not only structurally resemble neuronal MT (being enriched in Glu-tubulin) but also faithfully recapitulate the dynamics of MT in intact neurons. Apart from acting as a source neuronal tubulin, 25\% rat brain cytosol also provides the necessary MAPs to promote 

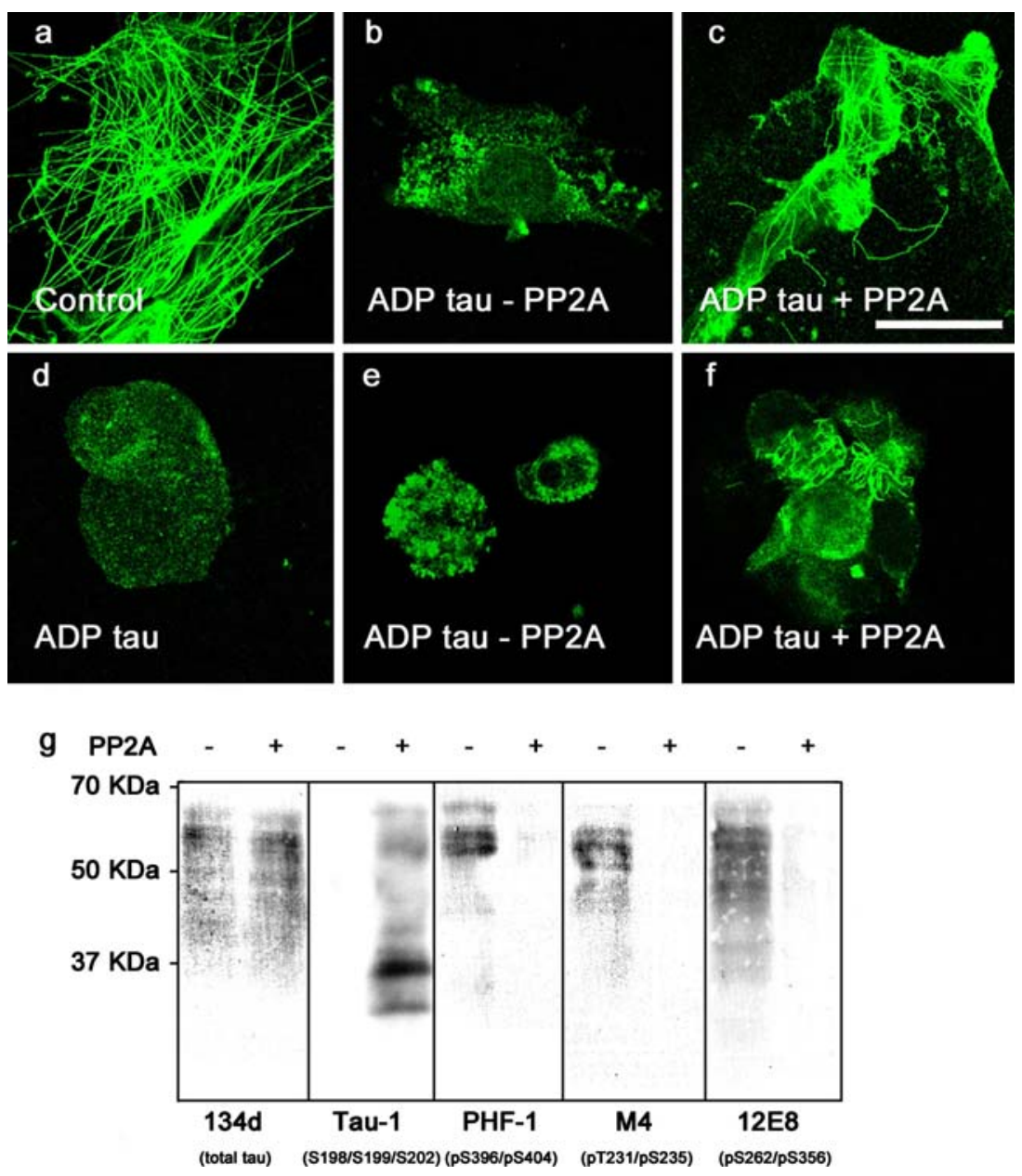

Fig. 7 Effect of dephosphorylation of AD P-tau on MT dynamics in Noco/Tri cells. AD P-tau was either treated with PP-2A prior to incubation for MT assembly (b, c) or during the incubation $(\mathbf{d}-\mathbf{f})$. In $\mathbf{b}, \mathbf{c}$ AD P-tau $(50 \mu \mathrm{g} / \mathrm{ml})$ was treated with PP-2A $(1 \mathrm{U} / \mathrm{ml})$ for $45 \mathrm{~min}$ at $37^{\circ} \mathrm{C}$ prior to its incubation with $25 \%$ rat brain cytosol for MT assembly in Noco/Tri cells; cells treated with the brain cytosol in the absence of AD P-tau were used as a control. Dephosphorylation of AD P-tau with PP-2A partially restored its activity to promote MT assembly (compare $\mathbf{c}$ with b). In $\mathbf{d}-\mathbf{f}, 25 \%$ rat brain extract was incubated with AD P tau $(50 \mu \mathrm{g} / \mathrm{ml})$ on Noco/Tri cells for $10 \mathrm{~min}$ to inhibit MT assem-

MT assembly. Under these conditions, MT assembly is seen as early as $1 \mathrm{~min}$ after incubation in the rat brain cytosol and the MT network so formed is stable for upto $4 \mathrm{~h}$. When rat brain cytosol is replaced by buffer, all MTs disassemble within $1 \mathrm{~h}$. Immunostaining of Noco/Tri cells shows that the assembled MTs closely resemble neuronal MT network. They are enriched in Glu-tubulin, which is found in stable assemblies of MT in differentiated cells. Although both MAP2 and tau co-stain with the MT network, MAP2 is the predominant MAP owing to its greater ability in promoting MT assembly [34]. When the reaction is carried out in the presence of AD P-tau, no MTs are seen, suggesting that AD P-tau inhibits MT assembly. Moreover, when AD P-tau is added to preassembled MT, all MT are bly (d). PP-2A was then added to the incubation mixture for $20 \mathrm{~min}$ before phosphatase inhibitors to inhibit PP-2A activity (f). Fresh GTP was supplied at this time and the cells were fixed after another $20 \mathrm{~min}$ incubation and stained with $\alpha$-tubulin antibody. MT partially "reassembled" in PP-2A treated Noco/Tri cells (f). PP-2A-catalyzed dephosphorylation of AD P-tau was confirmed by Western blot (g). AD P-tau $(50 \mu \mathrm{g} / \mathrm{ml})$ was treated with PP-2A $(1 \mathrm{U} / \mathrm{ml})$ for $45 \mathrm{~min}$ at $37^{\circ} \mathrm{C}$ and subjected to Western blot analysis. A decrease in tau phosphorylation at Tau-1, PHF-1, M4 and 12E8 epitopes is evident. Standard bar represents $25 \mu \mathrm{m}$

destroyed. The effect of AD P-tau on MT assembly is dose dependant, with concentrations of $50 \mu \mathrm{g} / \mathrm{ml}$ and above totally abolishing MT assembly. Normal tau in the brain cytosol, on the other hand, promotes MT assembly and additional unphosphorylated tau causes bundling of MTs. AD P-tau causes inhibition of MT assembly by sequestering normal tau and MAP2, as shown by co-staining of pSer422 immunoreactive tau (an epitope of AD P-tau) with Tau-1 immunoreactive tau (an epitope of normal tau), and with MAP2. When a protein phosphatase like PP-2A is introduced in the system, AD P-tau gets dephosphorylated and is now available for MT assembly. This is evidenced by observing MT "re-assembly" in the AD P-tau-treated Noco/Tri cells in which PP-2A has been added. 
In summary, AD P-tau inhibits MT assembly by a process, which involves sequestration of normal MAPs, including MAP2 and tau. This inhibitory activity is reversible, as MT re-assemble when normal tau becomes available following dephosphorylation of AD P-tau with PP-2A. Since tau is the major MAP associated with axonal MT whereas MAP2 is localized mainly in the dendritic pool of MT, removal of participating MAPs (tau and MAP2) from dynamic assemblies of MT forms, the basis of retrograde degeneration of both dendrites and axons seen in AD and related tauopathies. The Noco/Tri cells employed in this study provide a simple and relevant platform to study the neuronal cytoskeleton in a neuron-like environment. As a further extension, this model can be used to develop rapid screening assays for compounds with the potential to prevent or reverse neurofibrillary degeneration.

Acknowledgments We are grateful to Dr. Lester Binder, Northwestern University Medical School, Chicago, IL, USA for Tau-1 antibody; Dr. Peter Davies, Albert Einstein College of Medicine, Bronx, NY, for PHF-1 antibody; Dr. Dale Schenk, Elan Pharmaceuticals, San Francisco, CA, for 12E8 antibody; Dr. Yasuo Ihara, University of Tokyo, Tokyo, Japan, for M4 antibody; and Dr. A del C. Alonso, NYS Institute for Basic Research, Staten Island, NY, USA for anti-Glu-tubulin antibody. Janet Murphy provided secretarial assistance. Studies in our laboratories were supported in part by the New York State Office of Mental Retardation and Developmental Disabilities and NIH grant AG019158.

\section{References}

1. Alonso AC, Zaidi T, Grundke-Iqbal I, Iqbal K (1994) Role of abnormally phosphorylated tau in the breakdown of microtubules in Alzheimer disease. Proc Natl Acad Sci USA 91:5562-5566

2. Alonso AC, Grundke-Iqbal I, Iqbal K (1996) Alzheimer's disease hyperphosphorylated tau sequesters normal tau into tangles of filaments and disassembles microtubules. Nat Med 2:783-787

3. Alonso AC, Grundke-Iqbal I, Barra HS, Iqbal K (1997) Abnormal phosphorylation of tau and the mechanism of Alzheimer neurofibrillary degeneration: sequestration of microtubule-associated proteins 1 and 2 and the disassembly of microtubules by the abnormal tau. Proc Natl Acad Sci USA 94:298-303

4. Alonso AC, Zaidi T, Novak M, Grundke-Iqbal I, Iqbal K (2001) Hyperphosphorylation induces self-assembly of tau into tangles of paired helical filaments/straight filaments. Proc Natl Acad Sci USA 98:6923-6928

5. Alonso AC, Li B, Grundke-Iqbal I, Iqbal K (2006) Polymerization of hyperphosphorylated tau into filaments eliminates its inhibitory activity. Proc Natl Acad Sci USA 103:8864-8869

6. Benitez-King G, Tunez I, Bellon A, Ortiz GG, Anton-Tay F (2003) Melatonin prevents cytoskeletal alterations and oxidative stress induced by okadaic acid in N1E-115 cells. Exp Neurol 182:151-159

7. Brown S, Levinson W, Spudich JA (1976) Cytoskeletal elements of chick embryo fibroblasts revealed by detergent extraction. J Supramol Struct 5:119-130

8. Bulinski JC, Gundersen GG (1991) Stabilization of post-translational modification of microtubules during cellular morphogenesis. Bioessays 13:285-293
9. Cash AD, Aliev G, Siedlak SL, Nunomura A, Fujioka H, Zhu X, Raina AK, Vinters HV, Tabaton M, Johnson AB, Paula-Barbosa M, Avila J, Jones PK, Castellani RJ, Smith MA, Perry G (2003) Microtubule reduction in Alzheimer's disease and aging is independent of tau filament formation. Am J Pathol 162:1623-1627

10. Chen LQ, Wei JS, Lei ZN, Zhang LM, Liu Y, Sun FY (2005) Induction of Bcl-2 and Bax was related to hyperphosphorylation of tau and neuronal death induced by okadaic acid in rat brain. Anat Rec A Discov Mol Cell Evol Biol 287:1236-1245

11. Chohan MO, Haque N, Alonso AC, El-Akkad E, Grundke-Iqbal I, Grover A, Iqbal K (2005) Hyperphosphorylation-induced self assembly of murine tau: a comparison with human tau. J Neural Transm 112:1035-1047

12. Chohan MO, Khatoon S, Grundke-Iqbal I, Iqbal K (2006) Involvement of I2PP2A in the abnormal hyperphosphorylation of tau and its reversal by Memantine. FEBS Lett 580:3973-3979

13. Churcher I (2006) Tau therapeutic strategies for the treatment of Alzheimer's disease. Curr Top Med Chem 6:579-595

14. Dodart JC, Mathis C, Bales KR, Paul SM (2002) Does my mouse have Alzheimer's disease? Genes Brain Behav 1:142-155

15. Fath T, Eidenmuller J, Brandt R (2002) Tau-mediated cytotoxicity in a pseudohyperphosphorylation model of Alzheimer's disease. J Neurosci 22:9733-9741

16. Gilbert M, Fulton AB (1985) The specificity and stability of the triton-extracted cytoskeletal framework of gerbil fibroma cells. J Cell Sci 73:335-345

17. Gong CX, Singh TJ, Grundke-Iqbal I, Iqbal K (1994) Alzheimer's disease abnormally phosphorylated tau is dephosphorylated by protein phosphatase-2B (calcineurin). J Neurochem 62:803-806

18. Gong CX, Grundke-Iqbal I, Damuni Z, Iqbal K (1994) Dephosphorylation of microtubule-associated protein tau by protein phosphatase- 1 and $-2 \mathrm{C}$ and its implication in Alzheimer disease. FEBS Lett 341:94-98

19. Gong CX, Grundke-Iqbal I, Iqbal K (1994) Dephosphorylation of Alzheimer's disease abnormally phosphorylated tau by protein phosphatase-2A. Neuroscience 61:765-772

20. Grundke-Iqbal I, Iqbal K, Quinlan M, Tung YC, Zaidi MS, Wisniewski HM (1986) Microtubule-associated protein tau. A component of Alzheimer paired helical filaments. J Biol Chem 261:6084-6089

21. Grundke-Iqbal I, Iqbal K, Tung YC, Quinlan M, Wisniewski HM, Binder LI (1986) Abnormal phosphorylation of the microtubuleassociated protein tau (tau) in Alzheimer cytoskeletal pathology. Proc Natl Acad Sci USA 83:4913-4917

22. Gundersen GG, Bulinski JC (1986) Microtubule arrays in differentiated cells contain elevated levels of a post-translationally modified form of tubulin. Eur J Cell Biol 42:288-294

23. Hong HN, Yoon SY, Suh J, Lee JH, Kim D (2002) Differential activation of caspase- 3 at two maturational stages during okadaic acid-induced rat neuronal death. Neurosci Lett 334:63-67

24. Iqbal K, Alonso AC, Chen S, Chohan MO, El-Akkad E, Gong CX, Khatoon S, Li B, Liu F, Rahman A, Tanimukai H, Grundke-Iqbal I (2005) Tau pathology in Alzheimer disease and other tauopathies. Biochim Biophys Acta 1739:198-210

25. Iqbal K, Grundke-Iqbal I, Zaidi T, Merz PA, Wen GY, Shaikh SS, Wisniewski HM, Alafuzoff I, Winblad B (1986) Defective brain microtubule assembly in Alzheimer's disease. Lancet 23:421-426

26. Kopke E, Tung YC, Shaikh S, Alonso AC, Iqbal K, Grundke-Iqbal I (1993) Microtubule-associated protein tau: Abnormal phosphorylation of a non-paired helical filament pool in Alzheimer disease. J Biol Chem 268:24374-24384

27. Lindwall G, Cole RD (1984) Phosphorylation affects the ability of tau protein to promote microtubule assembly. J Biol Chem 259:5301-5305 
28. Liu F, Grundke-Iqbal I, Iqbal K, Gong CX (2005) Contributions of protein phosphatases PP1, PP2A, PP2B and PP5 to the regulation of tau phosphorylation. Eur J Neurosci 22:1942-1950

29. McNaught KS, Olanow CW (2006) Protein aggregation in the pathogenesis of familial and sporadic Parkinson's disease. Neurobiol Aging 27:530-545

30. Mesland DA, Spiele H (1984) Brief extraction with detergent induces the appearance of many plasma membrane-associated microtubules in hepatocytic cells. J Cell Sci 68:113-137

31. Montejo de Garcini E, Carrascosa JL, Correas I, Nieto A, Avila J (1988) Tau factor polymers are similar to paired helical filaments of Alzheimer's disease. FEBS Lett 236:150-154

32. Oyama F, Shimada H, Oyama R, Titani K, Ihara Y (1993) Betaamyloid protein precursor and tau mRNA levels versus beta-amyloid plaque and neurofibrillary tangles in the aged human brain. J Neurochem 60:1658-1664

33. Redeker V, Rossier J, Frankfurter A (1998) Posttranslational modifications of the $\mathrm{C}$-terminus of alpha-tubulin in adult rat brain: alpha 4 is glutamylated at two residues. Biochemistry 37:1483814844

34. Sandoval IV, Vandekerckhove JS (1981) A comparative study of the in vitro polymerization of tubulin in the presence of the microtubule-associated proteins MAP2 and tau. J Biol Chem 256:87958800
35. Schliwa M, van Blerkom J, Porter KR (1981) Stabilization and the cytoplasmic ground substance in detergent-opened cells and a structural and biochemical analysis of its composition. Proc Natl Acad Sci USA 78:4329-4333

36. Singh TJ, Haque N, Grundke-Iqbal I, Iqbal K (1995) Rapid Alzheimer-like phosphorylation of tau by the synergistic actions of non-proline-dependent protein kinases and GSK-3. FEBS Lett 358:267-272

37. Sloboda RD, Rudolph SA, Rosenbaum JL, Greengard P (1975) Cyclic AMP-dependent endogenous phosphorylation of a microtubule-associated protein. Proc Natl Acad Sci USA 72:177-181

38. Wang JZ, Grundke-Iqbal I, Iqbal K (1996) Restoration of biological activity of Alzheimer abnormally phosphorylated tau by dephosphorylation with protein phosphatase-2A, -2B and -1. Brain Res Mol Brain Res 38:200-208

39. Weingarten MD, Lockwood AH, Hwo SY, Kirschner MW (1975) Protein factor essential for microtubule assembly. Proc Natl Acad Sci USA 72:1858-1862

40. Yoon S, Choi J, Yoon J, Huh JW, Kim D (2006) Okadaic acid induces JNK activation, bim overexpression and mitochondrial dysfunction in cultured rat cortical neurons. Neurosci Lett 394:190-195 